
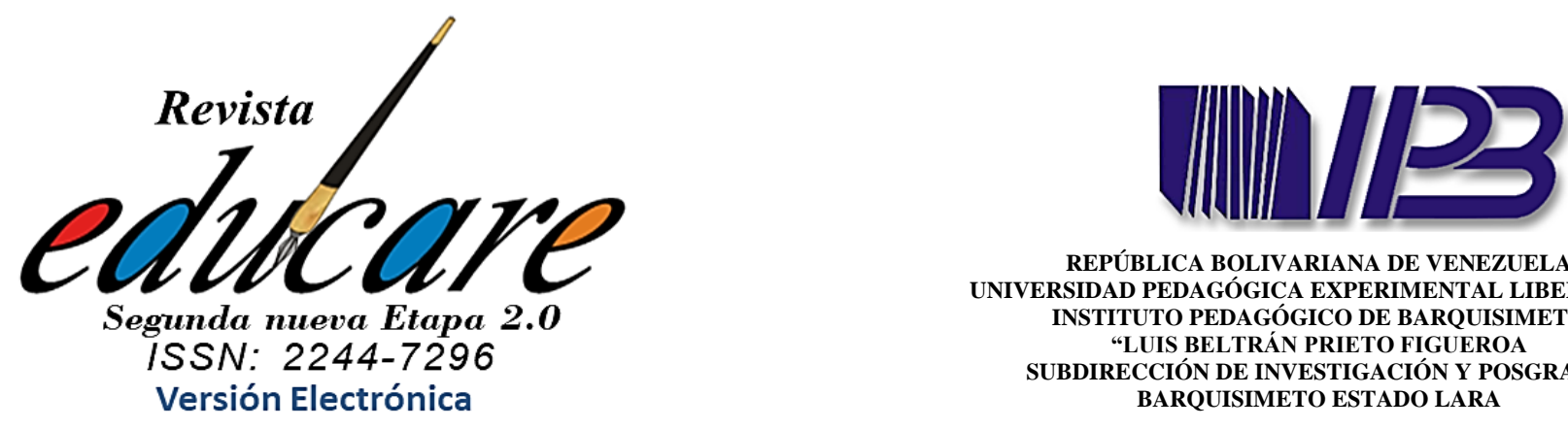

REPÚBLICA BOLIVARIANA DE VENEZUELA UNIVERSIDAD PEDAGÓGICA EXPERIMENTAL LIBERTADOR INSTITUTO PEDAGÓGICO DE BARQUISIMETO "LUIS BELTRÁN PRIETO FIGUEROA SUBDIRECCIÓN DE INVESTIGACIÓN Y POSGRADO BARQUISIMETO ESTADO LARA

Volumen 24 № 2 Mayo-Agosto 2020

(47-67)

Alirio Alejandro Tua Garcia*

ORCID: https://orcid.org/0000-0002-9240-0487

\section{PROYECTOS DE INVESTIGACIÓN CIENTÍFICA EN EDUCACIÓN MEDIA}

\author{
SCIENTIFIC RESEARCH PROJECTS IN \\ MIDDLE EDUCATION
}

Recibido:

17-02-2020

Aceptado:

17-06-2020 

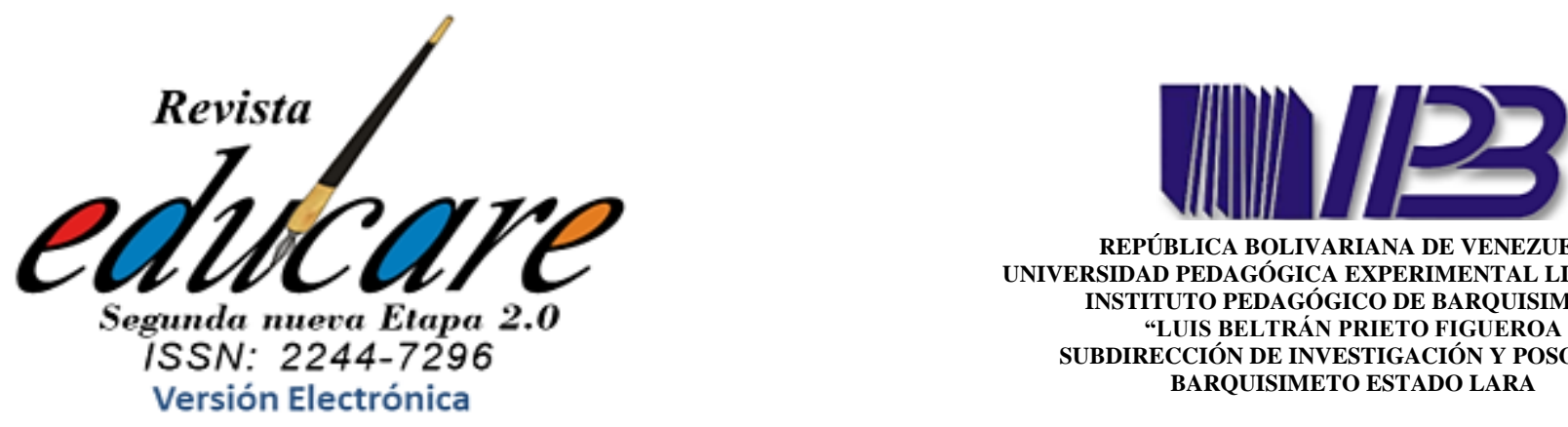

REPÚBLICA BOLIVARIANA DE VENEZUELA

UNIVERSIDAD PEDAGÓGICA EXPERIMENTAL LIBERTADOR

INSTITUTO PEDAGÓGICO DE BARQUISIMETO

"LUIS BELTRÁN PRIETO FIGUEROA

SUBDIRECCIÓN DE INVESTIGACIÓN Y POSGRADO BARQUISIMETO ESTADO LARA

Volumen 24 № 2 Mayo-Agosto 2020

$(47-67)$

\section{PROYECTOS DE INVESTIGACIÓN CIENTÍFICA EN EDUCACIÓN MEDIA}

SCIENTIFIC RESEARCH PROJECTS IN MIDDLE EDUCATION

\section{Resumen}

La investigación tuvo como objetivo diagnosticar la necesidad pedagógica de crear una guía didáctica referente a la elaboración del proyecto de investigación científica dirigida a los estudiantes de la Unidad Educativa Nacional San Francisco Javier en Barquisimeto, municipio Iribarren estado Lara. El estudio se apoyó en el paradigma positivista con un enfoque cuantitativo, sustentado en el nivel descriptivo, de campo, no experimental y transaccional. La población del estudio estuvo conformada por 210 estudiantes, a quienes se le aplicó un cuestionario impreso con escala tipo Likert de cinco alternativas de respuestas, validado mediante la técnica juicio de expertos y calculada su confiablidad con el coeficiente Alfa de Cronbach dando un resultado 0,96. Se concluyó que los estudiantes sujetos de estudio tienen una alta necesidad de poseer un guía didáctica que les permita la enseñanza de metodología de la investigación aplicada en educación.

Palabras clave: proyectos de investigación científica, educación media

\begin{abstract}
The objective of the research was to diagnose the pedagogical need to create a didactic guide regarding the preparation of the scientific research project aimed at students from the San Francisco Javier National Educational Unit in Barquisimeto, Iribarren municipality, Lara state. The study was based on the positivist paradigm with a quantitative approach, based on the descriptive, field, nonexperimental and transactional level. The study population consisted of 210 students, to whom a printed questionnaire with a Likert-type scale of five alternative responses was applied, validated using the expert judgment technique and calculated its reliability with the Cronbach's Alpha coefficient giving a result of 0 , 96. It was concluded that the students subject of study have a high need to have a didactic guide that allows them to teach applied research methodology in education.
\end{abstract}

Keywords: scientific research projects, secondary education 


\section{Introducción}

La investigación es una disciplina que surge por la inquietud del hombre para conocer la esencia de las cosas que le rodean e implica la búsqueda de nuevos conocimientos. De ahí, que es considerada una actividad humana inherente a todas las ramas del saber, puesto que por medio de ella se logra alcanzar la transformación y desarrollo de la educación en todos los niveles. Por consiguiente, unas de las razones de ser de la investigación es el crecimiento intelectual de las personas en cualquier campo del conocimiento y es una forma para promover el aprendizaje.

Por lo antes descrito, Arias (2012) menciona "la investigación implica la producción de un nuevo conocimiento, el cual puede estar dirigido a incrementar los postulados teóricos de una determinada ciencia (investigación pura o básica); o puede tener una aplicación inmediata en la solución de problemas prácticos" (p.22). Esto conlleva que la investigación científica se convierta en una exploración permanente de fenómenos que se pretende conocer utilizando métodos adecuados y precisos, en la búsqueda de ese conocimiento.

Es oportuno mencionar que, el campo educativo la investigación, tiene una gran influencia con el desarrollo socio económico del país. En tal sentido, la educación interviene en la formación y desarrollo de capacidades intelectuales en los estudiantes de diferente niveles académicos, el cual debe ser estimulado por parte de docentes investigadores para lograr alcanzar la producción de conocimiento en los educandos, como futuros profesionales y empresarios del país, por tal razón existen leyes que respalda la realización de la investigación científica en la educación.

Hay que destacar, en materia educativa, que la investigación está relacionada con todas las disciplinas y ciencias del saber. Al respecto, la Organización de las Naciones Unidas para la Educación, la Ciencia y la Cultura (UNESCO, 1998), plantea:

Promover, generar y difundir conocimientos por medio de la investigación y, como parte de los servicios que ha de prestar a la comunidad, proporcionar las competencias técnicas adecuadas para contribuir al desarrollo cultural, social y económico de las sociedades, fomentando y desarrollando la investigación científica y tecnológica a la par que la investigación en el campo de las ciencias sociales, las humanidades y las artes 
creativas. (p. 4)

En virtud a esto, la investigación es una estrategia en la educación para desarrollar conocimiento sistemático. Por tal razón, es difícil que el hombre pueda desligar la educación de la investigación. Al respecto, es trascendente lo planteado por Stenhouse (citado por Cerda, 1999) "la investigación no solo se debe reducir en producir mecánicamente un conjunto de conocimientos, sino que debe constituirse en un proceso válido para construir reconstruir, analizar, explorar, explicar, comprender y descubrir el conocimiento" (p. 18). De acuerdo a esto, la enseñanza de la investigación es importante porque concibe conocimiento para la transformación del sistema educativo.

Frente a esto, se considera que el educador y el alumnado son actores fundamentales de la educación, por tal razón, el docente en lugar de limitarse a transmitir información, debe crear el ambiente apropiado, para que el estudiante alcance la madurez y sea capaz de desarrollar sus propios ideales. Entre tanto, el compromiso de los docentes es transformar el pensamiento individual y tener una visión clara de la responsabilidad que tiene como mediador del aprendizaje. De igual manera, el docente investigador debe ocuparse de la articulación permanente de las competencias cognitivas del aprender a ser, a conocer, a hacer y a convivir.

No obstante, Delgado y Alfonzo (2019) afirman:

El papel docente-investigador implica reflexionar permanentemente, asumir una actitud crítica sobre lo aprendido en su formación y notificar saberes con sus pares $\mathrm{y}$ estudiantes, para establecer sinergias entre lo que se aprende e investiga, además sobre lo que se enseña y el para qué. Imparcialmente, la figura del docente-investigador lleva a considerar al sujeto como un representante reflexivo capaz de articular la praxis docente con la investigación. (p. 203-204)

Por ello, es primordial proporcionar al educando un papel de investigador con la orientación de sus profesores o expertos en el área de estudio. Por su parte, Freire (2004) expresa "no hay enseñanza sin investigación ni investigación sin enseñanza" (p. 17). Por lo cual, los estudiantes realizan proyectos de investigación científica desde la educación media en adelante. Por ello, es necesario que adquieran suficientes conocimientos en la elaboración de un 
proyecto con cientificidad, para lo cual deben aprender la metodología precisa y básica para emprender un estudio científico.

Con referencia a lo anterior, es impredecible garantizar la formación especializada a los estudiantes en el campo científico, humanístico, artístico, técnico y tecnológico, con el fin de que logren incorporarse exitosamente al proceso productivo social en la prosecución de sus estudios en la Educación Universitaria. Para avanzar hacia esas metas deben concluir sus estudios en educación media realizando el proyecto de investigación científica, requisito indispensable para el logro de su título como bachiller.

Es importante resaltar, que durante la formación en educación media los estudiantes adquieran competencias investigativa, Jaik (2013), refiere que son: "el conjunto de conocimientos, actitudes, habilidades y destrezas necesarias para llevar a cabo la elaboración de un trabajo de investigación” (p. 24). Cabe señalar que, las instituciones universitarias necesitan que los estudiantes posean habilidades investigativa, ya que tienen como objetivo ser centro de generación de investigación, como lo señala la Ley Orgánica de Educación (1980), en su Artículo 27:

Fomentar la investigación de nuevos conocimientos e impulsar el progreso de la ciencia, la tecnología, las letras, las artes y demás manifestaciones creadoras del espíritu en beneficio del bienestar del ser humano, de la sociedad y del desarrollo independiente de la nación. (p.13)

Resulta oportuno decir, en metodología existe diversidad de métodos y forma de percibir a la investigación, por lo tanto, existe muchas disyuntivas en cuanto a la elaboración de proyecto, puesto que, los investigadores especialistas en diversas áreas del conocimiento adoptan posturas metodológicas con el fin de conseguir una producción científica. Piedra y Martínez (2007), enfatizan:

La producción científica es la parte materializada del conocimiento generado, es más que un conjunto de documentos almacenados en una institución de información. Contempla todas las actividades académicas y científicas de un investigador. Este fenómeno se encuentra ligado a la mayoría de los acontecimientos en los que se ven involucradas las personas, atendiendo al resultado de los trabajos de 
investigación e innovación. (p. 33)

En atención a lo anterior, la producción científica es una actividad de investigación realizada en las instituciones educativas y tiene como unos de sus fines dar a conocer información de un tema en estudio. Dichas actividades las define Flores, Meléndez, y Mendoza (2019), como aquellas que: "están relacionadas con la producción, difusión y aplicación de conocimientos científicos y tecnológicos" (p. 68). Estas acciones deben estar presentes en todos los investigadores, la difusión de los resultados es parte final de la investigación científica.

En este contexto, los estudiantes de quinto año en educación media realizan proyectos de investigación científica para optar al título de bachiller, tal es el caso concreto de la Unidad Educativa Nacional San Francisco Javier de Barquisimeto, municipio Iribarren Estado Lara; al ser consultados sobre su realización expresan: no sé cómo hacerlo, requiero un material didáctico para guiarme, no tengo quien me oriente. De igual forma, manifiestan que los profesores no detallan con propiedad sobre cómo se elabora los proyectos de investigación y que no consiguen información actualizada.

En entrevistas informales con algunos estudiantes manifiestan: carecemos de un recurso que nos permita elaborar el proyecto de investigación que nos exigen para optar a nuestro título de bachiller, necesitamos una material que nos permita conocer sobre metodología, he observado que ningún docente del área de proyecto tiene una guía que nos facilite alcanzar conocimiento de investigación y las normativas, es difícil hacer unos objetivos de investigación sin una referencia.

Al mismo tiempo, en conversaciones informales agregan los docentes de proyecto que les gustaría contar con una guía para ejecutar actividades inherentes a métodos y normativas científicas. Adicional declaran, en quinto año se debe tener una especie de recurso didáctico para desarrollar más eficiente la enseñanza-aprendizaje en la investigación. A su vez, verbalizan que les gustaría poseer un material actualizado que ayude a los estudiantes a comprender los pasos de una producción intelectual.

Así que, en la referida institución educativa de educación media se observan escenarios que parecieran revelar la necesidad pedagógica que tienen los estudiantes de quinto año en la elaboración de una guía didáctica referente a la elaboración del proyecto de 
investigación, puesto que en las palabras de los estudiantes y docente no existe un recurso que oriente al alumnado en el desarrollo de sus producciones científica.

En ocasiones desde la experiencia empírica del autor es inevitable utilizar una guía didáctica y especializada acorde a la realidad de los contenidos dictado para adquirir un conocimiento de manera innovadora, donde se estipule actividades con un lenguaje adecuado a los estudiantes de acuerdo a su nivel educativo, para obtener un efectivo proceso de aprendizaje en determinada área de estudio.

Sobre estas consideraciones, se estableció como objetivo general de la investigación: Diagnosticarla necesidad pedagógica de crear una guía didáctica referente a la elaboración del proyecto de investigación científica dirigida a los estudiantes de la Unidad Educativa Nacional San Francisco Javier en Barquisimeto, municipio Iribarren estado Lara.

\section{Marco teórico}

La teoría del aprendizaje significativo de Ausubel (1973) sostiene que los conceptos previamente formados o descubiertos por el alumno en su entorno son la base para la adquisición de nuevos conceptos; de ahí la importancia de los conocimientos previos, según el autor:

Un aprendizaje es significativo cuando los contenidos son relacionados de modo no arbitrario y sustancial (no al pie de la letra) con lo que el alumno ya sabe. Por relación sustancial y no arbitraria se debe entender que las ideas se relacionan con algún aspecto existente específicamente relevante de la estructura cognoscitiva del alumno, como una imagen, un símbolo ya significativo, un concepto o una proposición. (p. 18)

Esto quiere decir, que en el proceso educativo, es importante considerar lo que el individuo posee conocimiento de tal manera que establezca una relación con aquello que debe aprender. Este proceso tiene lugar porque el estudiante tiene en su estructura cognitiva conceptos, estos son: ideas, proposiciones, estables y definidos, con los cuales la nueva información puede interactuar.

Entre los enfoques que sustentan el aprendizaje significativo está el constructivismo. A tal efecto, Papalia (1988) parte del postulado: 
El conocimiento no se descubre, se construye. Básicamente puede decirse que el constructivismo es el modelo que mantiene que una persona, tanto en los aspectos cognitivos, sociales y afectivos del comportamiento, no es un mero producto del ambiente ni un simple resultado de sus disposiciones internas, sino una construcción propia que se va produciendo día a día como resultado de la interacción de estos dos factores. (p. 175)

Desde la posición constructivista, el conocimiento no es una copia de la realidad sino una construcción del ser humano. Esta construcción se realiza a partir de los esquemas que la persona ya posee. Lo anteriormente expuesto, es fundamental para el presente estudio, ya que las acciones de índole educativo crean contextos que influyen en los procesos de aprendizaje, razón por la cual este estudio se fundamenta en el diagnóstico de necesidad pedagógica para crear una guía didáctica referente a la elaboración del proyecto de investigación científica

\section{Guía Didáctica}

Una Guía Didáctica es un recurso que facilita el proceso de aprendizaje, orientando al estudiante, en las actividades academias. Este material proporciona al docente una herramienta muy útil para lograr que el alumno desarrolle habilidades y destrezas en cualquier asignatura. En efecto, Aguilar (2018) señala que una guía didáctica "es el material educativo que deja de ser auxiliar, para convertirse en herramienta valiosa de motivación y apoyo; pieza clave para el desarrollo del proceso de enseñanza" (p. 183).

De igual manera, la guía didáctica es un material que facilita la comprensión de los contenidos a través de la información teórica y actividades práctica, debido a que, permiten reforzar cada sesión de clases. Zambrano (2007) define "una guía didáctica como un recurso impreso con orientación técnica para el estudiante" (p. 33). Este recurso propicia la transferencia del conocimiento, por medio de actividades interactivas entre el docente y los estudiantes.

Las guías didácticas son un recurso creados para fortalecer el aprendizaje didáctico con orientaciones. En referencia a esto, García Aretio (1997) las define como: 
Un recurso para el apoyo a la docencia que, en general no sustituye sino que acompaña a un texto base. Desde el punto de vista de la enseñanza sirve de acompañamiento y complementa el texto cuando este último no está adaptado a la enseñanza (p. 97).

De este modo, la guía didáctica es un recurso de apoyo para facilitar el aprendizaje de manera didáctica y es considerado un recurso con pautas a seguir para enseñar de forma significativa. Asimismo, Bruner (1999) señala: "la necesidad de diseñar guía didáctica, impresa o en formatos digital, con el propósito de que se convierta en el andamiaje que posibilite al estudiante avanzar con mayor seguridad en el aprendizaje autónomo” (p. 58). Es decir, una guía didáctica como recurso permite que el estudiante avance y sea agente activo de su propio aprendizaje, por consiguiente el recurso mencionado le proporcionará seguridad para consolidar su proceso de aprendizaje y formación en la investigación científica.

\section{Investigación científica en educación media}

La investigación es la búsqueda del conocimiento y consiste en indagar acerca de los aspectos relacionados con un hecho a estudiar y surge de la necesidad que tiene el hombre en conocer la esencia de las cosas. En la Universidad Pedagógica Experimental Libertador, Instituto Pedagógico Luis Beltrán Prieto Figueroa (UPEL-IPB), el Documento Base del Currículo de la UPEL (2011) menciona:

La investigación como eje curricular, se convierte en la vía para articular; la formación, capacitación, actualización, ampliación, profundización y perfeccionamiento, constituye el pilar fundamental para la formación de los profesionales e investigadores de la docencia y áreas afines. De allí que sea necesaria la preparación en el área investigativa desde diferentes paradigmas, mediante la creación de escenarios propicios, así como la previsión de planes, programas y proyectos cónsonos con la realidad. (p. 31)

En esa misma línea, Sabino (2002) expresa: "la investigación se originan en las ideas, sin importar que tipo de paradigma fundamente nuestro estudio ni el enfoque que habremos de seguir" (p. 30). Paralelamente, la investigación está siempre vinculada a la realidad, a la búsqueda del saber, en el campo de la ciencia, la tecnología, la sociedad; su función principal 
es la obtención de nuevos conocimientos, partiendo de dudas e inquietudes planteadas por el hombre.

La investigación científica es un proceso sistemático, que se centra en la producción de conocimientos, mediante la creación de nuevas teorías. Generalmente la investigación científica surge de ideas, interrogantes e hipótesis que se plantea el hombre. Para Martínez (2006), define la investigación: "como un proceso de construcción de conocimiento, de indagación, de interrogación requiere, así mismo, la construcción de sujetos que indaguen y se interroguen, que se expongan y se arriesguen en esa irracionalidad insegura que es la ciencia" (p. 86). Por tal motivo, este proceso implica la resolución de problemas planteados tanto en la educación como en y todas las ramas del saber.

Cabe mencionar que, en todo trabajo de investigación, el autor se forma una idea sobre un tema o problema. Por lo cual, procede a recopilar información en relación al estudio que se desea realizar en diferentes fuentes impresa y digital, contribuyendo a enriquecer los conocimientos que surgen a medida que se realiza una exploración; además le permitirá formular hipótesis, realizar experimentos, recopilar datos y finalmente, hacer conclusiones encontrando respuestas a las preguntas formuladas.

Por otra parte, el proyecto de investigación científica es el trabajo que se elabora con el fin de llevar a cabo los procedimientos necesarios para la realización de la investigación, por ende, se puede decir que este trabajo será el plan a seguir para lograr los objetivos propuestos por el investigador. Los proyectos de investigación según Barrios, Reyes y Muñoz (2009) consideran "actualmente una herramienta para indagar necesidades o problemas concretos que se traducen en alternativas de solución, bienes o servicios” (p. 232).

En esta perspectiva, Rodríguez (2016), plantea "los docentes que administran unidades curriculares de Proyectos: tienen responsabilidades administrativas y gerenciales enmarcadas hacia la descentralización, la aplicación del Proceso de Aprendizaje Socio Integrador Productivo" (p. 28). En relación a lo anteriormente expuesto, Valles (2017) afirma:

El proyecto de una investigación es el documento que expresa detalladamente el problema a tratar, así como su importancia, justificación de resolverlo, significado para la ciencia en general o para una especialidad en particular, los antecedentes encontrados en las fuentes de información, el propósito u objetivos, las hipótesis, la metodología a 
usar, las referencias utilizadas. (p. 13)

En este orden de ideas, se observa claramente, que el proyecto que realizan los estudiantes de cualquier nivel académico debe contener toda la información propia de la investigación, esbozando los pasos que sigue el método científico. Una vez que se realiza el trabajo, se busca difundir los hallazgos o resultados encontrados durante el proceso de investigación, para ello se realizan actividades, tales como la presentación pública del estudio ante un jurado acreditado, los docentes de la institución, familiares y público en general.

Es importante decir que, los proyectos de investigación, trabajo de grado, tesis o cualquier producción científica son una herramienta de los centros educativos que le permite al estudiante optar a un título, tal es el caso de los estudiantes de la Unidad Educativa Nacional San Francisco Javier en Barquisimeto, municipio Iribarren estado Lara, donde tienen que realizar un proyecto de investigación científica como requisito último para optar al título de bachiller.

\section{Estructura para elaborar un proyecto de investigación}

En término de Bisquerra (2009) la elaboración de un proyecto de investigación científica, se deben desarrollar los siguientes puntos:

1. Planteamiento del problema: Generalmente se basa en una situación problemática presente en determinado lugar. La observación, las entrevistas y conversaciones formal o informal son clave para desarrollar el planteamiento. En esta sección se desarrollan tres elementos: preguntas de investigación, objetivos de investigación y justificación del estudio.

2. Marco referencial o teórico: Para comenzar a elaborar este apartado es necesario detectar, obtener y consultar la literatura impresa como digital para así extraer la información de interés. El mismo está conformado por: antecedentes del estudio, base teórica, fundamentación o base legal.

3. Marco metodológico: En esta sección se describe como se realizó la investigación atendiendo a la metodología de cada estudio de acuerdo a los objetivos. Este se define el tipo de investigación, el diseño, la modalidad de estudio, Sistema de Variable, población, técnicas e instrumento de información, validez, confiabilidad, 
técnicas de análisis de datos y procedimiento de la investigación.

4. Resultados: Es la redacción de los datos encontrados sobre el asunto investigado utilizando por lo general la estadística descriptiva con tablas y gráficos. Esto último depende de la metodología, la técnica e instrumento utilizado por el investigador.

5. Conclusiones: Se sintetiza los resultados arrojados por el estudio de acuerdo a los objetivos establecidos.

6. Recomendaciones: Son sugerencia que se le hace a los involucrados de forma directa o indirecta en el estudio.

7. Referencias: Es el aparatado donde se encuentra reflejado los datos de cada bibliográfica impresa o digital utilizada dentro de la investigación científica y se configura de acuerdo a las normas del centro adscrito el investigador.

8. Anexos: Son aquellos documentos que apoyan el estudio y estos sustenta la elaboración de la investigación.

Cabe resaltar que, la estructura de los proyectos de investigación depende de la institución académica donde están registrados el o los autores. Aunado a esto, hay que tomar en cuenta el enfoque del estudio, sus parámetros y la normativa por el cual se rige el centro educativo o la universidad que acredite la producción científica.

\section{Aspectos Metodológicos}

\section{Naturaleza de la Investigación}

El presente estudio está enmarcado bajo el paradigma positivista con enfoque cuantitativo de acuerdo al objetivo de la investigación. En referencia a lo anterior, Hernández, Fernández y Baptista (2014) afirman que el enfoque cuantitativo "usa la recolección de datos para probar hipótesis, con base en la medición numérica y el análisis estadístico, para establecer patrones de comportamiento y probar teorías" (p. 4). Dado en este caso, los datos recolectados fueron procesados a través de la estadística descriptiva para realizar la debida interpretación de la información recolectada. 


\section{Nivel y Diseño de la Investigación}

En función al objetivo de la investigación se enmarca en un nivel descriptivo, los autores anteriormente citados establecen: "los estudios descriptivos buscan especificar las propiedades, las características y los perfiles importantes de personas, grupos, comunidades o cualquier otro fenómeno que se someta a análisis” (p. 92).

En relación con el diseño se sustentó en una investigación de campo, ya que se recolectaron datos sin realizar manipulación de los datos o de la variable en estudio. Además, se ubica en una investigación de carácter no experimental transaccional para Toro (2006), “es la que se realiza sin manipular en forma deliberada ninguna variable" (p. 158)

\section{Población y Muestra}

Sobre la población objeto de estudio estuvo conformada por 210 estudiantes de quinto año de la Unidad Educativa Nacional "San Francisco Javier." Al ser una población de fácil acceso para el investigador se seleccionó toda la población de estudio por consiguiente la muestra es censal, Zarcovich (2005) menciona que esta muestra logra "datos de todas las unidades del universo acerca de las cuestiones, bloques, que constituyen el objeto del censo. Los datos se recogen en una muestra que representa el total del universo" (p. 78).

\section{Técnica e Instrumento de Recolección de Información}

Se implementó la técnica de encuesta y como instrumento un cuestionario impreso en escalamiento que consiste, según Hernández y otros (2014), en un "conjunto de ítems que se presentan en forma de afirmaciones para medir la reacción del sujeto en tres, cinco o siete categorías" (p. 245). Para su construcción se utilizó una escala tipo Likert de cinco alternativa de respuestas: Alta Necesidad (AN), Mediana Necesidad (MN), Baja Necesidad (BN), Muy Baja Necesidad (MBN), No es Necesidad (NEN).

Posteriormente, el instrumento elaborado se le determinó su validez y confiabilidad. Hernández y otros (2014) mencionan "La validez es el grado en que realmente un instrumento mide la variable que pretende medir" (p. 280). Es decir, la validez de contenido permitió a través de juicio de expertos emitir opiniones sobre la claridad, pertinencia y congruencia de 
cada ítem.

Para Arias (2012): "la confiabilidad es el grado con el cual el instrumento prueba su consistencia, por los resultados que produce al aplicarlo repetidamente al objeto de estudio" (p. 81). La confiabilidad se realizó mediante el coeficiente Alfa de Cronbach a través del software estadístico SPSS, por lo cual se aplicó una prueba piloto a diez estudiantes en el Liceo Bolivariano "Ezequiel Bujanda" en Barquisimeto. Estos mantienen la misma característica que la población en estudio, los cuales dieron su consentimiento para la recolección de los datos, obteniéndose el valor de 0,96. Significa que el instrumento se ubica dentro del rango de confiabilidad muy alta según la escala por Ruiz Bolívar (2002). (Ver cuadro 1). En otras palabras, el instrumento mide lo que desea medir. En este caso, la necesidad pedagógica de crear una guía didáctica referente a la elaboración del proyecto de investigación científica dirigida a los estudiantes de la Unidad Educativa Nacional "San Francisco Javier."

El autor antes citado indica que la confiabilidad "está enfocada como el grado de homogeneidad de los ítems del instrumento en relación con la característica que pretende medir" (p. 44). Los valores de la confiablidad oscilan entre 0 y 1 , donde cero significa nula o que el instrumento no es confiable y uno que representa máxima confiabilidad.

\section{Cuadro 1}

Interpretación de la magnitud del Coeficiente de Confiabilidad de un instrumento

\begin{tabular}{|cc|}
\hline Rangos & Magnitud \\
\hline 0,81 a 1,00 & Muy Alta \\
0,61 a 0,80 & Alta \\
0,41 a 0,60 & Moderada \\
0,21 a 0,40 & Baja \\
0,01 a 0,20 & Muy Baja \\
\hline
\end{tabular}

Nota: Tomado de Ruiz Bolívar (2002)

Por otra parte, Larios (1999) sostiene "una variable es cualquier característica, fenómeno, proceso, hecho, ser o situación susceptible de ser objeto de estudio y de indagación en una investigación" (p. 65). La variable del presente estudio es la necesidad pedagógica de crear una guía didáctica referente a la elaboración del proyecto de investigación científica dirigida a los estudiantes de la Unidad Educativa Nacional San Francisco Javier. La misma se conceptualiza como la carencia o ausencia de un recurso didáctico que no posee el 
alumnado para realizar su producción científica.

Para el cumplimiento del objetivo planteado en este estudio, se siguieron los siguientes pasos:

1. Detección del problema.

2. Revisión bibliográfica.

3. Redacción del planteamiento de problema.

4. Elaboración de: antecedentes, base teóricas y fundamentación legal.

5. Estructura del marco metodológico.

6. Diseño del instrumento de recolectar los datos.

7. Validación del instrumento.

8. Consentimiento Informado para la obtención de los datos.

9. Aplicación de pruebas pilotos.

10. Verificación de la Confiabilidad del instrumento.

11. Organización de los resultados.

12. Análisis y discusión de los resultados.

13. Formulación de la conclusión y recomendaciones.

\section{Presentación de Resultados}

A continuación, se presentan los resultados del estudio analítico de investigación a través de la información recogida en el instrumento de recolección de datos dirigido a los doscientos diez (210) estudiantes de quinto año de la Unidad Educativa Nacional San Francisco Javier en Barquisimeto, municipio Iribarren estado Lara, quienes conformaron la muestra de estudio y que dieron su consentimiento para participar en el estudio. Los datos obtenidos, mediante un conteo manual fueron organizados en tablas.

Los resultados se calcularon en porcentajes e ilustrados a través de tablas representativas de las respuestas promedios de cada dimensión. Éstos, a su vez, fueron interpretados y confrontados con algunos de los contenidos de las bases teóricas, a modo de configurar la estructura sobre la cual se formularon las conclusiones. 


\section{Tabla 1}

Frecuencias absolutas y porcentajes. Dimensión: Recurso Didáctico - Indicador: Necesidad pedagógica.

\begin{tabular}{|c|c|c|c|c|c|c|c|c|c|c|c|}
\hline \multirow{3}{*}{$\mathbf{N}^{\circ}$} & \multirow{3}{*}{$\begin{array}{l}\text { Exprese el grado de necesidad, } \\
\text { que usted tiene de contar con una } \\
\text { guía didáctica para la elaboración } \\
\text { del proyecto de investigación } \\
\text { científica que le permita: }\end{array}$} & \multicolumn{10}{|c|}{ Alternativa de respuestas } \\
\hline & & \multicolumn{2}{|c|}{$\mathbf{A N}$} & \multicolumn{2}{|c|}{$\mathbf{M N}$} & \multicolumn{2}{|c|}{$\mathbf{B N}$} & \multicolumn{2}{|c|}{ MBN } & \multicolumn{2}{|c|}{ NEN } \\
\hline & & $\mathbf{F}$ & $\%$ & $\mathbf{F}$ & $\%$ & $\mathbf{F}$ & $\%$ & $\mathbf{F}$ & $\%$ & $\mathbf{F}$ & $\%$ \\
\hline 1 & $\begin{array}{l}\text { Realizar discusiones soci } \\
\text { sobre metodología de invest }\end{array}$ & 2 & 96 & 10 & 4 & 0 & 0 & 0 & 0 & 0 & 0 \\
\hline 2 & $\begin{array}{l}r \text { actividades fund } \\
\text { dología. }\end{array}$ & 21 & 100 & 0 & 0 & 0 & 0 & 0 & 0 & 0 & 0 \\
\hline 3 & $\begin{array}{l}\text { ejemplo de cada estructura } \\
\text { royecto de investigación. }\end{array}$ & 21 & 100 & 0 & 0 & 0 & 0 & 0 & 0 & 0 & 0 \\
\hline 4 & $\begin{array}{l}\text { Aclarar aspectos formales para la } \\
\text { presentación de normativas de } \\
\text { investigación. }\end{array}$ & 2 & 100 & 0 & 0 & 0 & 0 & 0 & 0 & 0 & 0 \\
\hline & valores rromedios & 20 & 99 & 3 & 1 & 0 & 0 & 0 & 0 & 0 & \\
\hline
\end{tabular}

Nota: AN =Alta Necesidad, $\mathrm{MN}=$ Mediana Necesidad, BN=Baja Necesidad, $\mathrm{MBN}=$ Muy Baja Necesidad, NEN =No es Necesidad.

Se procede a detallar la data obtenida en la dimensión recurso didáctico, se aprecia lo siguiente en la tabla 1, el ítem1, 96\% de los estudiantes encuestados evidenciaron alta necesidad en adquirir una guía para la elaboración del proyecto de investigación científica que le permita realizar discusiones socializadas sobre metodología de investigación, mientras que $4 \%$ expresan mediana necesidad.

En el ítem 2 se desprende que 100\% de los encuestados consideran alta necesidad de contar con una guía didáctica que le permita ejecutar actividades fundamentada en metodología. En cuanto al ítem 3,100\% de los estudiantes consideran que es alta necesidad una guía didáctica que permita ilustrar ejemplo de cada parte de un proyecto de investigación.

En relación con el ítem4, 100\% de los estudiantes manifestaron que la necesidad es alta en cuanto al poseer una guía didáctica que permita aclarar aspectos formales para la presentación de normativas de investigación. 
De allí los valores promedios obtenidos son los siguientes: $99 \%$ en la escala de alta necesidad y $1 \%$ en la alternativa mediana necesidad. La sumatoria de las respuestas indica que los estudiantes encuestados necesitan la guía didáctica para la elaboración de proyectos científicos. Al respecto, Flor (1997) señala "los recursos didácticos son útiles y aprovechado para favorecer el aprendizaje de los alumnos y es el docente quien lo administra en cualquier área del saber” (p.17).

\section{Tabla 2}

Frecuencias absolutas y porcentajes. Dimensión: Producción científica - Indicador: Investigación.

Exprese el grado de necesidad, que usted tiene de contar con una

Alternativa de respuestas
AN MN BN MBN NEN

$N^{\circ}$ guía didáctica para la

elaboración del proyecto de

investigación científica que le $\begin{array}{lllllllllll}\text { F } & \% & \text { F } & \% & \text { F } & \% & \text { F } & \% & \text { F } & \%\end{array}$ permita:

5 Comprender los métodos de $\begin{aligned} & \text { Comprigación } \\ & \text { investio }\end{aligned}$

investigación

$6 \begin{aligned} & \text { Visualizar la estructura de } \\ & \text { planteamiento de problema. }\end{aligned} \quad \begin{array}{lllllllllll}195 & 92 & 10 & 8 & 0 & 0 & 0 & 0 & 0 & 0\end{array}$

$7 \begin{aligned} & \text { Entender los lineamientos de un } \\ & \text { proyecto con rigor científico. }\end{aligned} \quad \begin{array}{lllllllllll}210 & 100 & 0 & 0 & 0 & 0 & 0 & 0 & 0 & 0\end{array}$

8 La enseñanza de metodología de la

investigación aplicada en $\begin{array}{lllllllllll}210 & 100 & 0 & 0 & 0 & 0 & 0 & 0 & 0 & 0\end{array}$ educación.

Valores Promedios

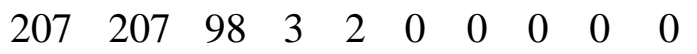

Nota: AN =Alta Necesidad, MN=Mediana Necesidad, BN=Baja Necesidad, MBN= Muy Baja Necesidad, NEN = No es Necesidad.

Respecto a los datos expuestos en la tabla 2, se destaca que el ítem 5, 100\%, de los encuestados expresan que tiene una alta necesidad en poseer una guía didáctica para comprender los métodos de investigación.

En cuanto al ítem 6,92\% de los estudiantes consideran alta necesidad obtener una guía didáctica que permita visualizar la estructura de un planteamiento de problema, al mismo tiempo $8 \%$ de los estudiantes expresan mediana necesidad.

A su vez, en el ítem 7,100\% de los encuestados afirman tener alta necesidad de una guía didáctica para entenderlos lineamientos de un proyecto con rigor científico. En el ítem 8 , 
$100 \%$ de los encuestados aseveran que tiene alta necesidad de una guía que permita la enseñanza de metodología de la investigación aplicada en educación.

Los promedios obtenidos son los siguientes: $98 \%$ de los estudiantes de quinto año considera alta necesidad de poseer una guía didáctica para la enseñanza y elaboración del proyecto de investigación, mientras $2 \%$ opinan que es mediana necesidad. Los resultados obtenidos coinciden con lo expresado por Stenhouse (1987) quien enfatiza "el docente debe desarrolla una enseñanza basada en la investigación" (p. 89).

\section{Conclusiones}

Al diagnosticar la necesidad pedagógica de crear una guía didáctica referente a la elaboración del proyecto de investigación científica dirigida a los estudiantes de la Unidad Educativa Nacional San Francisco Javier en Barquisimeto, municipio Iribarren estado Lara, se concluye que los sujetos de estudio tienen una alta necesidad de poseer un guía didáctica que les permita la enseñanza de metodología de la investigación aplicada en educación, visualizar la estructura de un planteamiento de problema, ilustrar ejemplo de cada estructura de un proyecto de investigación y aclarar aspectos formales para la presentación de normativas de investigación, puesto que, se evidenció en los encuestados debilidad en el área de investigación, trayendo consigo dificultades en la construcción de su producción científica.

\section{Recomendaciones}

De acuerdo a los resultados obtenidos en la presente investigación se recomienda:

-A los docentes establecer parámetro de investigación para todos los estudiantes de quinto año. Se les invita a los docentes a diseñar una guía didáctica que oriente la elaboración de los proyectos de investigación científica. Al momento de construir el recurso didáctico realizar la validación tanto por los docentes que dictan los contenidos en proyecto de investigación como la de estudiantes en el uso del mismo lo que permitiría saber que el recurso puede ser utilizado por los actores educativos antes mencionados.

-De esta manera, se sugiere manipular la guía didáctica en formato impreso como digital y que este contenga enlaces web relacionados con la investigación para obtener un 
aprendizaje cónsono a sus necesidades. Además, se les propone a los docentes investigadores a construir un programa de capacitación para desarrollar competencias investigativas donde los participantes sean estudiantes de quinto año de la institución donde se originó la investigación.

\section{Referencias}

Aguilar, E. (2018). Aprendizaje, competencias y rendimiento. Madrid: La Muralla.

Arias, F. (2012). El proyecto de investigación: Introducción a la metodología científica. Caracas, Venezuela: Episteme.

Ausubel, D (1973). Psicología Educativa: Un punto de vista cognoscitivo. México: Editorial Trillas. Segunda Edición.

Barrios, Z., Reyes, L., y Muñoz, D. (2009). Desarrollo de competencias a través de proyectos de investigación. Revista Telos, 11(2), 229-243. http://www.redalyc.org/articulo.oa?id=99312517007

Bisquerra, R. (2009). Metodología de la investigación educativa. Editorial: La Muralla S.A. https://www.academia.edu/38170554/METODOLOG\%C3\%8DA_DE_LA_INVESTIG ACI\%C3\%93N_EDUCATIVA_RAFAEL_BISQUERRA.pdf

Bruner, J. (1999). Escuela para pensar: Una ciencia de aprendizaje en el aula. $\quad$ Madrid Paidos.

Cerda, H. (1999). La investigación Formativa en el Aula. La Pedagogía como Investigación. Bogotá: Cooperativa Editorial Magisterio.

Delgado, Y. y Alfonzo, R. (2019). Competencias Investigativas del Docente Construidas durante la Formación Universitaria. Revista Scientific, 4(13), 200-220. https://doi.org/10.29394/Scientific.issn.2542-2987.2019.4.13.10.200-220

Flor, J. (1997). Recursos didácticos para la investigación en el aula. Sevilla. Diada editorial S.L

Flores, E., Meléndez, J., y Mendoza, R. (2019). Producción científica como medio para la transformación social desde las universidades. Revista Scientific, 4(14), 62-84. https://doi.org/10.29394/Scientific.issn.2542-2987.2019.4.14.3.62-84

Freire, P. (2004). Pedagogía de la Autonomía. Editorial: Siglo XXI de España Editores. 
García

Aretio,

E.

(1997).

Guía

Instruccional.

http://www.sensei.ieec.uned.es/miguel/tesis/noe32.html

Hernández, R; Fernández, C. y Baptista, P. (2014). Metodología de la investigación. México: McGraw-Hill.

Jaik, A. (2013). Competencias Investigativas: Una mirada a la Educación Superior [Tesis de doctorado no publicada]. Universidad Pedagógica Experimental Libertador (UPEL), Barquisimeto

Larios (1999). Algo de Metodología https://www.uv.es/webgid/Descriptiva/32_variables.html

Ley Orgánica de Educación (1980). Gaceta Oficial de la República de Venezuela $N^{\circ}$ 2635 (Extraordinario), 26 de julio de 1980.

Martínez, M. (2006). La nueva ciencia. México: Trillas.

Papalia, D. (1988). Psicología Educativa. México: McGrawHill.

Piedra, Y., y Martínez, A. (2007). Producción Científica. Revista Ciencias de la Información, 38(3), 33-38. https://www.redalyc.org/pdf/1814/181414861004.pdf

Rodríguez, J. (2016). Redimensión del proceso de aprendizaje para la transformación de los Institutos y Colegios Universitarios en Venezuela, [Tesis de doctorado de la $\begin{array}{lll}\text { Universidad de Carabobo] } & \end{array}$ http://mriuc.bc.uc.edu.ve/bitstream/handle/123456789/4116/jrodriguez.pdf?sequence=1

Ruiz Bolívar, C. (2002). Instrumentos de investigación educativa. Procedimientos para su diseño y validación. Venezuela: CIDEG.

Sabino, C. (2002). El Proyecto de Investigación Científica. Caracas: Panapo.

Stenhouse, L. (1987).La Investigación como Base a la Enseñanza. Madrid, España: Ediciones Morata.

Toro, I. (2006). Método y conocimiento: Metodología de la investigación. México: Fondo.

UNESCO (1998). Declaración Mundial sobre la Educación Superior en el siglo XXI: Visión y Acción. París, Francia: Organización de la Naciones Unidas para la Educación, la Ciencia y la Cultura. https://unesdoc.unesco.org/ark:/48223/pf0000113878_spa 
UPEL

$$
\text { (2011). Documento }
$$

Base

del

Currículo

de

la

UPEL.

http://geminis.upel.edu.ve/do/Repositorio/TransformacionCurricular/DOCUMENTO.pd $\underline{f}$

Valles,

E.

(2017)

Estrategia

educativa.

https://www.ucn.edu.co/recurso\%20Cemav/documento/Aprendizaje.

Zambrano, A. (2007). Didáctica, pedagogía critica y saber. Bogotá. Colombia Cooperativa.

Editorial Magisterio

Zarcovich, P. (2005). Metodología de la investigación. México: McGraw-Hill. 